\title{
A Model Study of Electrical Impedance Tomography Using Synthetic Finite Precision Data
}

\author{
R. Buchert and K. Schilcher \\ Institut für Physik, Johannes-Gutenberg-Universität, Staudingerweg 7, D-55099 Mainz, Germany \\ Z. Naturforsch. 49a, 920-926 (1994); received April 15, 1994 \\ We use a resistor network model for electrical impedance reconstruction from boundary data. On \\ the basis of a particular choice of currents we construct a stable inversion algorithm.
}

\section{Introduction}

Electrical impedance tomography (EIT) attempts to reconstruct the conductivity distribution inside a body from current and voltage measurements on its boundary [1]. Concerning medical applications [2] EIT constitutes a promising method for safe in vivo monitoring of morphology and physiological processes by relatively simple instrumentation, i.e. at low costs. Unfortunately the problem of inversion of the boundary data is much more severe than in alternative imaging systems such as X-ray tomography $[3,4]$. The problem of calculating internal resistances from boundary data is a nonlinear one, and, in addition, extremely ill-posed [3]. Small errors in the boundary data will result in non-controllably large errors in the output resistances. Mathematically this instability arises from the unboundedness of the (existing) inverse of the derivative of the mapping $\sigma \rightarrow P_{\sigma}$ from the space of conductivities $\sigma$ (in a given domain) to the space of corresponding Dirichlet-to-Neuman mappings $P_{\sigma}$ [4]. The inverse problem of inferring $\sigma$ by measuring $P_{\sigma}$ must be regularized [5].

For practical applications involving the conductivity distribution of a body one must introduce an appropriate discretisation. The simplest one just replaces the body by a resistance network, which is of course more or less equivalent to subdividing the body into small voxels (pixels) of constant conductivity. Making a number of simplifying physical assumptions such as validity of quasistatic conditions, absence of electrical sources within the body, scalar conductivity (not tensor) and validity of Ohm's law (conductivity indepen-

Reprint requests to Prof. Dr. K. Schilcher, Institut für Physik, Universität Mainz, Staudingerweg 7, D-55099 Mainz. dent of the current density) Maxwell's equations just reduce to Kirchhoff's laws [1]. It is interesting to observe how the results of the classical boundary value problems of electrodynamics translate into discrete matrix statements $[6,7]$. In particular, it was shown by Curtis and Morrow [7] by means of an explicit algorithm that the internal resistors can be uniquely determined from boundary current and voltage data.

In this paper we propose and test a method of solving the two-dimensional inverse EIT problem that makes use of some of the results of [7]. The emphasis is on adapting the design of the EIT measurement configuration so as to optimize the condition of the corresponding inverse problem and to minimize the time of numerical reconstruction. The condition of the inverse problem is directly related to the number of unknowns in the discrete non-linear system. Employing the adapted currents proposed by Curtis and Morrow, the number of unknowns is reduced so dramatically that current conservation can be used directly for the reconstruction of the conductivities. In this way the repetitive time consuming solution of the foreward problem, which is conventionally employed in EIT is avoided altogether. The non-linear system thus obtained is solved by a regularized LevenbergMarquardt algorithm [8]. This algorithm is widely used in the solution of non-linear equations, but the detailed method of solution is of secondary importance here in comparison to the problem of the choice of the optimal current configuration.

To simulate an experimental situation we determine the Curtis-Morrow currents numerically from onesource one-sink synthetic data by solving a linear system of equations. We study the sensitivity of our algorithm to degraded input data. It is not surprising that, if the errors are confined to the Curtis-Morrow currents great improvement is observed. 
There is a very substantial literature on the object of EIT. For an extensive list see two recent review monographs [9]. We refer in our paper only to those references of direct relevance.

\section{The Curtis-Morrow Algorithm}

For simplicity we consider a quadratic network defined by an $n \times n$ lattice with equidistant lattice points $(i, j)$, which therefore can be assumed to have integer coordinates $i, j=0,1, \ldots, n-1$. Conductivities $S$ are associated with links between lattice points as shown in Fig. 1 for $n=6$.

The currents and voltages in the network are governed by Kirchhoff's first rule (charge conservation), i.e. at a given knot the sum of the incoming currents is zero. Using Ohm's law, this rule reads in our case

$$
\begin{aligned}
& S[(i, j),(i+1, j)]\left\{\phi_{I}(i+1, j)-\phi_{I}(i, j)\right\} \\
& +S[(i, j),(i, j+1)]\left\{\phi_{I}(i, j+1)-\phi_{I}(i, j)\right\} \\
& +S[(i-1, j),(i, j)]\left\{\phi_{I}(i-1, j)-\phi_{I}(i, j)\right\} \\
& +S[i, j-1),(i, j)]\left\{\phi_{I}(i, j-1)-\phi_{I}(i, j)\right\}=0, \\
& i, j=1,2, \ldots, n-2
\end{aligned}
$$

at the interior points $(i, j), i, j=1,2, \ldots, n-2$. In addition, there are the equations

$$
\begin{array}{r}
S[(i, 0),(i, 1)]\left\{\phi_{I}(i, 0)-\phi_{I}(i, 1)\right\}=I(i, 0), \\
i=1,2, \ldots, n-2
\end{array}
$$

specifying the generated Neuman boundary conditions at the boundary points $(i, 0), i=1,2, \ldots, n-2$, at the bottom of the network and analogously for all other boundary points (see Figure 2). Here $I(i, 0)$ is the applied current entering the network at the boundary point $(i, 0)$, and $\phi_{I}(i, j)$ is a corresponding potential at node $(i, j)$. Although the totality of (1), (2) (for all considered current configurations) is linear with respect to the conductivities, it represents a set of non-linear equations with respect to all unknows, i.e. with respect to the conductivities and interior potentials.

The first step towards an explicit solution of this system of equations is the choice of suitable current configurations for which the potentials at the boundary nodes are to be measured. Curtis and Morrow prove the remarkable theorem that for each boundary point $(i, 0), i=1, \ldots, n-2$ at the bottom of the network there exists a current configuration $I_{(i, 0)}$ that can be applied at the boundary nodes with non-
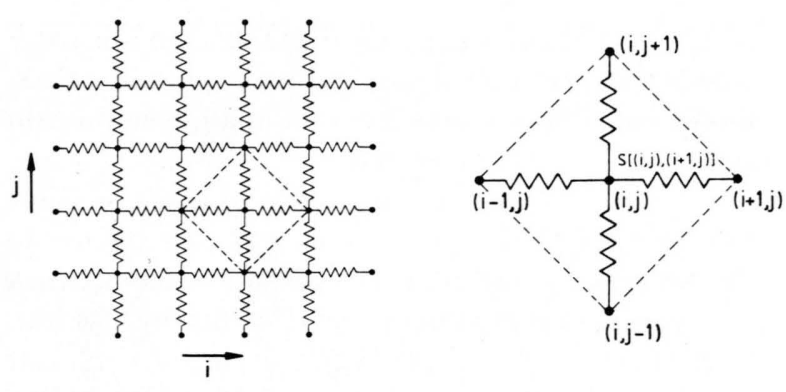

Fig. 1. Assignment of conductivities in the network.

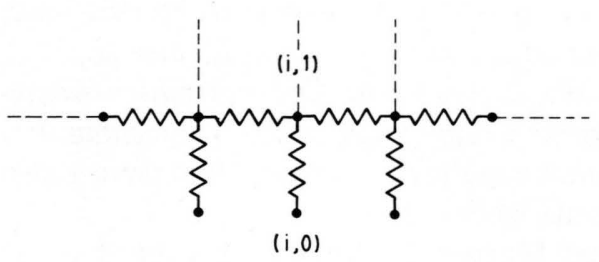

Fig. 2. Boundary points of the network.

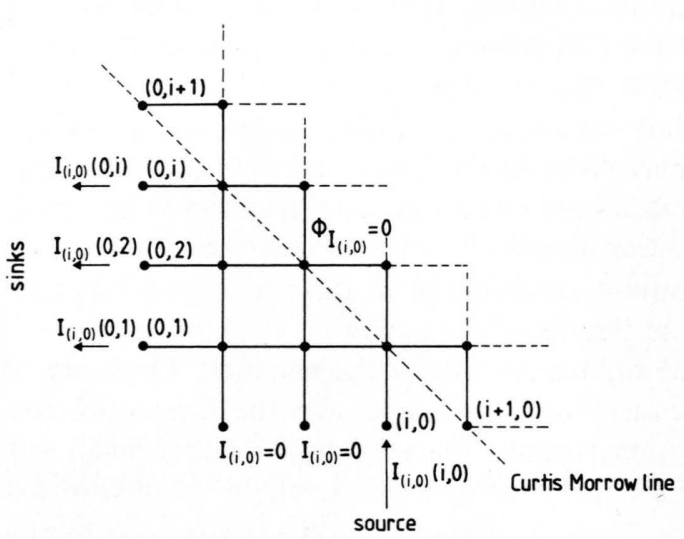

Fig. 3. Curtis-Morrow current configuration $I_{(i, 0)}$.

vanishing currents only at $(i, 0)$ and $(0, j), j=1, \ldots, i$, such that the corresponding potential $\phi_{I_{(i, 0)}}$ is constant on and above the diagonal connecting the nodes $(i+1,0)$ and $(0, i+1)$ (see Figure 3$)$. We call these configurations Curtis-Morrow currents (CM currents) and the corresponding diagonals Curtis-Morrow lines (CM lines). Fixing the arbitrary additive constant, which appears also in the discrete formulation of the Neumann problem, the constant can be chosen to be zero. It goes without saying that this theorem is not restricted to the lower left corner of the resistor network but applies to the other three corners as well. It 
is worth noting that there is no analogue to this theorem in the continuous case, where a potential (harmonic function) which is constant in an open domain must be identically constant.

It is not difficult to see that the CM currents allow to inductively find the conductance of each resistor in the network by starting at the corners of the network and working towards the center, CM line by CM line. There is no need to solve large systems of nonlinear equations. In this way Curtis and Morrow showed that the discrete EIT problem as defined above has a unique solution [7]. To determine an $n \times n$ network requires only $2(n-2) \mathrm{CM}$ currents to be measured, for example $I_{(i, 0)}, i=1,2, \ldots, n-2$, and $I_{(n-1, j)}$, $j=n-2, n-3, \ldots, 1{ }^{1}$ Using CM currents is somewhat analogous to applying the Gauß algorithm to a system of linear equations; to some extent there is also some triangularization here.

Curtis and Morrow furthermore proved that the a priori unknown $\mathrm{CM}$ currents can be obtained by linear combination of current configurations with one source and one sink only. For definiteness consider again the CM current $I_{(i, 0)}$ at the lower left corner. It can be written as a linear combination of the $i$ configurations with a single current source at $(i, 0)$ and a single current sink at $(0,1), \ldots,(0, i)$, respectively, with the coefficients being uniquely determined by a normalization condition and the requirement that the potential corresponding to this combination is constant at the boundary nodes $(n-1, j), j=1, \ldots, n-2$, on the right hand side of the network. Therefore, in practice, at each inductive step the Curtis-Morrow algorithm requires the solution of rather small systems of linear equations (maximally $n-2$ unknowns). The electronic equipment to realize current configurations with one source and one sink only is still rather manageable.

The Curtis-Morrow model/algorithm can be easily implemented with a computer algebra language like REDUCE. Unfortunately, one finds that this approach is severely ill-conditioned, which of course only reflects the fact that the original problem in the continuum is extremely ill-posed. The explicit solution is actually useless for practical problems except for

\footnotetext{
1 Since there are $4(n-2)-1$ linearly independent boundary currents it seems that here only half of the information encoded in the Dirichlet-Neumann mapping is needed to uniquely determine the conductivities. This is not really true, since in addition one uses current conservation.
}

very small networks or unrealistically small data errors.

In this paper we propose and test an iterative Levenberg-Marquardt approach which is regularized essentially by the requirement of positivity. We have to consider (1) and (2) for all current configurations simultaneously, so that unfortunately we are faced with a rather large system of nonlinear equations. The main advantage of using $\mathrm{CM}$ currents is then that this drastically reduces the number of unknowns and produces a lot of zeros in the Jacobi matrix of the nonlinear system. It is only due to this fact that employing current conservation directly for the reconstruction makes sense, at least for the relatively small lattices of EIT. If required, the low dimensional linear systems relating the $\mathrm{CM}$ currents to the actually measured one source-one sink currents can be easily regularized à la Tikhonov-Phillips or by cutting the singular value decomposition.

\section{Stabilization}

The reconstruction of a resistance network from boundary voltages and currents is a typical ill-conditioned problem. Small changes in the (measured) input data can produce very large changes in the output resistances. Useful information may nevertheless be extracted if the system of equations is regularized. Of the various methods available we choose a LevenbergMarquardt prescription [8].

The non-linear system to be solved and regularized is of the form

$$
A x+y^{T} B x+i=0,
$$

where $x$ is a column vector whose first $2(n-2)(n-1)$ elements are the unknown conductivities while the rest of the elements are zero, $y$ is a column vector whose first $2(n-2)(n-1)$ elements are zero and the following elements are the unknown interior potentials, $A$ is a matrix "linear" in the measured boundary potentials and $B$ is a matrix containing only zeros and ones.

Although, as mentioned above, $2(n-2) \mathrm{CM}$ currents suffice to uniquely fix the explicit solution, we bring into play all $\mathrm{CM}$ currents. ${ }^{2}$ According to the

\footnotetext{
2 Employing only the $2(n-2) \mathrm{CM}$ currents corresponding to opposite corners would have the advantage of decoupling the equations into two non-linear systems, each involving only half of the unknown conductivities.
} 


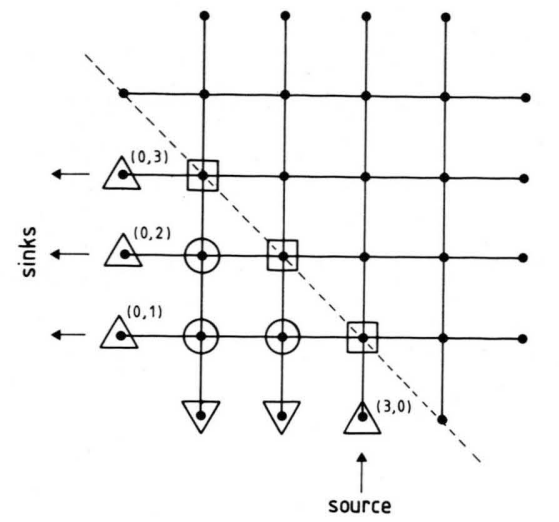

Fig. 4. Determination of Curtis-Morrow currents.

number of boundary nodes there are $4(n-2)$ of them. The equations employed with each measurement are shown exemplary for $I_{(3,0)}$ in Figure 4. The nodes marked with $\Delta$ contribute equations specifying Neumann boundary conditions (2) and the nodes marked with $\bigcirc$ or $\square$ contribute equations expressing current conservation (1). The $\square$-equations which express current conservation along Curtis-Morrow lines are redundant, but we found that including them improved our results significantly. At the boundary electrodes marked with $\nabla$ which carry no current we set the potential of the unique nearest interior neighbour equal to the boundary potential right away. The interior potentials on the CM lines are set to zero, so they do not appear in our equations. In summary, we have to perform $4 \frac{(n-2)(n-1)}{2}$ measurements with appropriate one source - one sink current configurations to determine $4(n-2) \mathrm{CM}$ current configurations, which according to the scheme indicated in Fig. 4 yield

$$
4\left(\frac{(n-2)(n-1) n}{6}+\frac{(n-2)(n+1)}{2}\right)
$$

nonlinear equations in altogether

$$
2(n-2)(n-1)+4 \frac{(n-4)(n-3)(n-2)}{6}
$$

unknowns, $2(n-2)(n-1)$ conductivities and

$$
4 \frac{(n-4)(n-3)(n-2)}{6}
$$

unknown interior potentials. These numbers fix the dimensions of the quantities in (3). In Table 1 we give
Table 1.

\begin{tabular}{lrrrrll}
\hline$n$ & 4 & 5 & 6 & 7 & 10 & $n \rightarrow \infty$ \\
\hline \# unknown conductivities & 12 & 24 & 40 & 60 & 144 & $2 n^{2}$ \\
\# unknown interior potentials & 0 & 4 & 16 & 40 & 224 & $2 / 3 n^{3}$ \\
\# equations & 36 & 76 & 136 & 220 & 656 & $2 / 3 n^{3}$ \\
\# unknown interior potentials/ & 0 & $1 / 6$ & $2 / 5$ & $2 / 3$ & $112 / 77$ & $n / 3$ \\
\# unknown conductivities & & & & & \\
\# equations/\# unknowns & 3 & $19 / 7$ & $17 / 7$ & $11 / 5$ & $41 / 23$ & 1 \\
\hline
\end{tabular}

the ratio of the number of unknown interior potentials to the number of conductivities and the ratio of the number of equations to the number of unknowns for different values of $n$. Obviously these ratios become worse with increasing $n$ showing that using current conservation directly for the reconstruction rather than integrating out the unknown interior potentials by considering powers [5] is restricted to relatively small lattices as the ones expected to be used in clinical EIT.

The system (3) is well suited for the Levenberg-Marquardt method [8] as the Jacobian can be explicitly calculated. The Levenberg-Marquardt method is a maximum neighbourhood method which performs an optimal interpolation between the gradient method and the Newton-Raphson method for solving systems of nonlinear algebraic equations, the interpolation being based upon the maximum neighbourhood in which the truncated Taylor series is a valid approximation for the nonlinear system.

Stability is achieved by the requirement of positivity. This can be implemented very easily by an appropriate prescription for the repetitive adjustment of the parameter $\lambda$ in the Levenberg-Marquardt method. In addition to the standard strategy proposed in the original papers [8] we make sure that at each iterative step $\lambda$ is sufficiently large so as to keep all conductivities positive. As compared to positivity, controlling the number of iterations plays a minor role for the regularization.

\section{Results}

As described in Sects. II and III we have employed a two step procedure based on the Curtis-Morrow algorithm and Levenberg-Marquardt regularization to determine the conductivities of a resistance network from boundary data under realistic error conditions. We considered the specific case of an $n=6$ resis- 


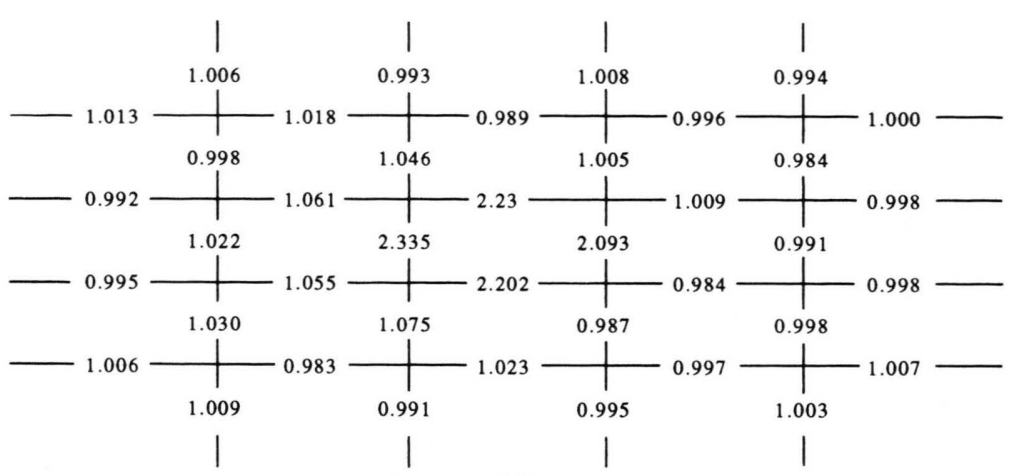

(a)

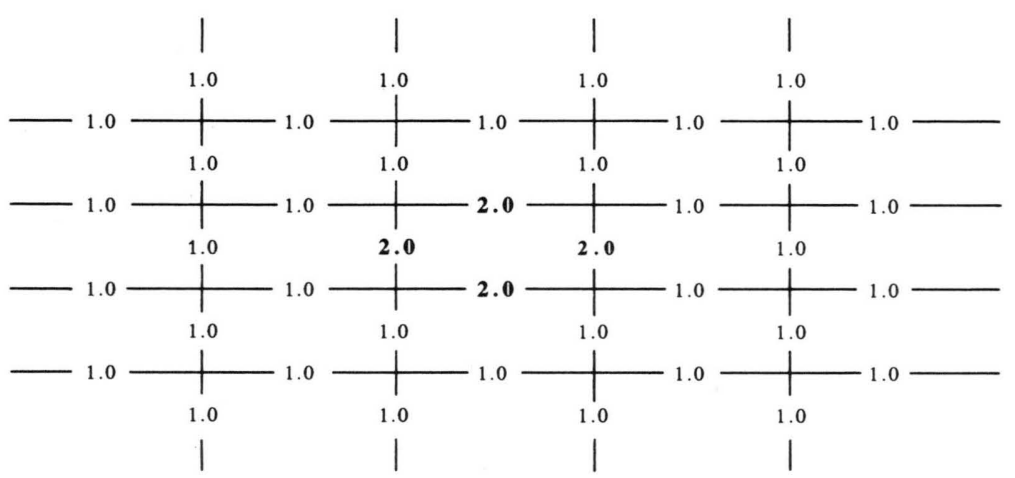

(b)

tance lattice involving 40 unknowns resistances (see Figure 1).

As a first step, we produce synthetic boundary data assuming a given resistance configuration, i.e. we solve the stable forward Neumann problem. We keep only the boundary data (currents and voltages) and then try to reconstruct the resistances after having added various errors to these synthetic data.

As an initial test of the method we employ exact data (to computer accuracy) and then try to reconstruct the resistances. Allowing for sufficiently many iterations the method reproduces the assumed resistances to very high accuracy, as expected. From the point of view of the computer accuracy (15 decimals) the resistance lattice is still very small and the ill-conditionedness of the problem does not manifest itself yet. These results only serve to check the consistency of our method (and the correctness of the FORTRAN program), and details need not be given here.

To mimic realistic experiments we degrade the synthetic data with random errors of different magnitude.
Fig. 5. Image reconstruction after 6 iterations (a) with actual conductances as shown in (b). The relative error in the one source - one sink input currents and voltages was taken to be $10^{-4}$. The Levenberg-Marquardt process was initialized with all conductances set equal to 1 (and corresponding interior potentials, see text).
The results may be described as follows: To reconstruct the resistances in this network, the error on the one source - one sink boundary data should be $\leq 10^{-4}$. In Fig. 5 we give the result for the reconstruction of a central resistance pattern in self-explanatory matrix form (compare Figure 1). The determination of the CM currents was not regularized. The required accuracy is probably too high for any impedance tomographic application as the network by itself constitutes only a model discretisation to the continuous conductivity of human tissues, and a rough network therefore automatically introduces sizeable errors.

The situation changes dramatically if the error is only imposed on the CM currents introduced in Section II. We find that even with $1 \%$ errors the resistances can be reconstructed both qualitatively and quantitatively. Results are given in Figure 6.

In both cases we initialized the Levenberg-Marquardt process with all conductances set equal to 1 . Inserting this value (or any arbitrary values) for the conductances in (3), the resulting system is just linear 


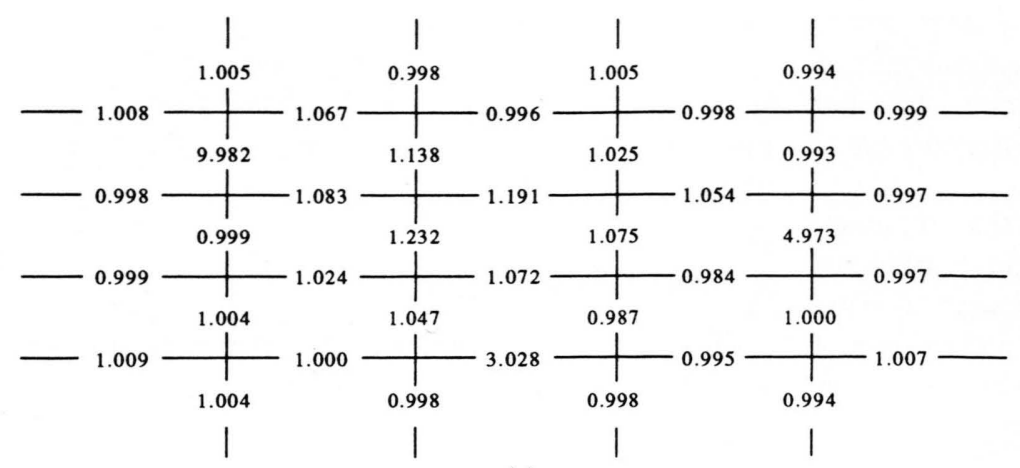

(a)

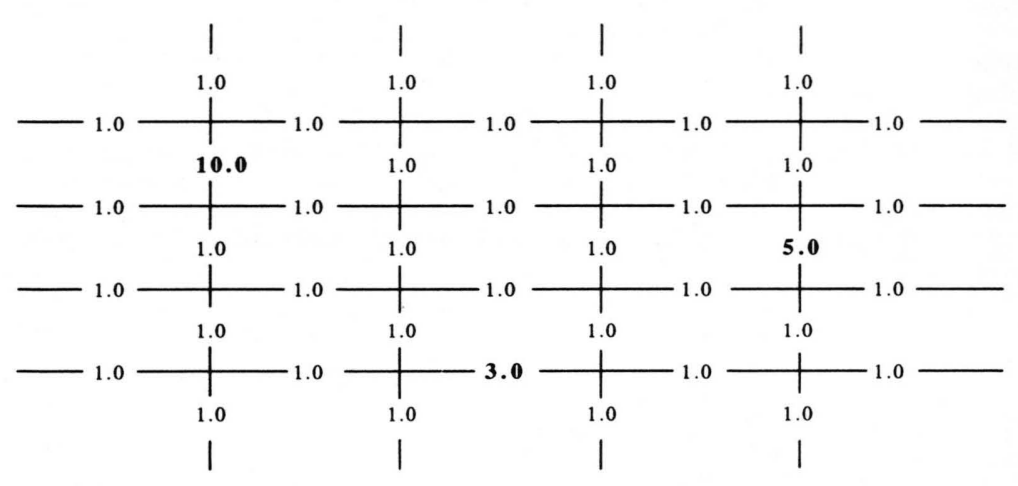

Fig. 6. Image reconstruction after 30 iterations (a) with actual conductances as shown in (b). The relative error in the Curtis-Morrow currents and the corresponding voltages was taken to be $10^{-2}$. The Levenberg-Marquardt process was initialized with all conductances set equal to 1 (and corresponding interior potentials, see text).

(b)

in the unknown interior potentials. The Moore-Penrose solution of this system was used to fix the starting values of the interior potentials.

It remains to be shown how the CM currents can be practically introduced into impedance tomography improving experiment design. It is conceivable to first measure the one source - one sink configurations, to compute the CM currents within the network model and then to actually apply these currents to the object under consideration. Some fine tuning will be necessary to optimally meet the CM conditions. Investigations in this direction are under way.

When considering large lattices, the interior potentials should be integrated out by using the electric power which relates boundary data to interior data in the reconstruction. This requires a solution of the forward Neumann problem at each iterative step. It can be seen rather easily, however, that this is also greatly simplified for the CM currents, and in particular it does not require the time consuming solution of large linear systems. Furthermore, once the foreward problem has been solved, the computation of the power is also very fast since only a small number of conductivities contribute. For the same reason the resulting (non-linear) system is almost diagonal, i.e. is of good condition.

Within the present approach it is not difficult to employ more general, non-rectangular two-dimensional networks [7]. However, there is still the important step from tomographic to real three dimensional imaging to do. This requires three dimensional networks, where unfortunately things become somewhat more involved. The transfer of the main concepts, such as the CM currents, however, is rather straightforward.

Furthermore it remains to avoid the simplifying assumptions mentioned in the introduction. Which of them are most restrictive depends on the particular application. By now there are very powerful algorithms for the numerical forward solution of the 
complete Maxwell equations (time dependent, tensor $\varepsilon$ and $\mu$ ) originally developed for applications in high energy physics [6]. At present we examine to what extent these algorithms can be used for the purpose of electrical impedance imaging.

Another problem to be dealt with is that of modeling the boundary shape of a given body within the used discretisation with sufficient accuracy. To some extent this problem can be solved by making use of conformal mappings. In two dimensions and under

[1] A. D. Seagar, D. C. Barber, and B. H. Brown, IEE Proceedings 134, 201 (1987).

[2] B. H. Brown, D. C. Barber, and A. D. Seagar, Clin. Phys. Physiol. Meas. 6, 109 (1985).

[3] W. R. Brecken and M. K. Pidcock, in: Proceedings of the XVIth Workshop of Interdisciplinary Study of Inverse Problems: Some Topics of Inverse Problems (P. C. Sabatier, ed.), Montpellier, Nov. 30 - Dec. 4, 1987, pp. $254-$ 264.

[4] A. K. Louis, Inverse und schlecht gestellte Probleme, Teubner Studienbücher Mathematik, Teubner, Stuttgart 1989. A. K. Louis, Inverse Problems 8, 709 (1992). quasistatic conditions the relevant equation reads $\operatorname{div}(\sigma \operatorname{grad} \phi)=0$ (current conservation). Evidently this equation is invariant with respect to conformal mappings. We will discuss this point in more detail elsewhere.

\section{Acknowledgement}

One of us (K.S.) would like to thank G. and O. Schuhfried for numerous discussions.

[5] W. R. Breckon and M. K. Pidcock, Clin. Phys. Physiol. Meas. 8, Suppl. A, 77 (1987).

[6] M. Bartsch et al., Computer Physics Communications 72, 22 (1992).

[7] E. B. Curtis and J. A. Morrow, SIAM J. Appl. Math. 50, 918 (1990).

[8] K. Levenberg, Quart. Appl. Math. 2, 164 (1944); D. W. Marquardt, J. Soc. Indust. Appl. Math. 11, 431 (1963).

[9] E. Holder, ed. Electrical Impedance Tomography, University College London Press; J. G. Webster, ed. Electrical Impedance Tomography, Adam Hilger, Bristol 1990. 